\title{
Significant Racial Differences in the Key Factors Associated with Early Graft Loss in Kidney Transplant Recipients
}

\author{
David J. Taber ${ }^{a}$ Kevin Douglass ${ }^{c}$ Titte Srinivas $^{b}$ John W. McGillicuddy ${ }^{a}$ \\ Charles F. Bratton $^{a}$ Kenneth D. Chavin ${ }^{a}$ Prabhakar K. Baliga ${ }^{a} \quad$ Leonard E. Egede $^{d}$ \\ Divisions of ${ }^{a}$ Transplant Surgery and ${ }^{b}$ Transplant Nephrology, ${ }^{c}$ College of Medicine, and ${ }^{d}$ Center for Health Disparities \\ Research, Medical University of South Carolina, Charleston, S.C., USA
}

\section{Key Words}

Kidney transplantation · African-American · Graft loss ·

Cardiovascular disease

\begin{abstract}
Background: There is continued and significant debate regarding the salient etiologies associated with graft loss and racial disparities in kidney transplant recipients. Methods: This was a longitudinal cohort study of all adult kidney transplant recipients, comparing patients with early graft loss $(<5$ years) to those with graft longevity (surviving graft with at least 5 years of follow-up) across racial cohorts [AfricanAmerican (AA) and non-AA] to discern risk factors. Results: 524 patients were included, $55 \%$ AA, 151 with early graft loss (29\%) and 373 with graft longevity (71\%). Consistent within both races, early graft loss was significantly associated with disability income [adjusted odds ratio (AOR) $2.2,95 \% \mathrm{Cl} 1.1$ 4.5], Kidney Donor Risk Index (AOR 3.2, 1.4-7.5), rehospitalization (AOR 2.1, 1.0-4.4) and acute rejection (AOR 4.4, 1.711.6). Unique risk factors in AAs included Medicare-only insurance (AOR 8.0, 2.3-28) and BK infection (AOR 5.6, 1.3-25). Unique protective factors in AAs included cardiovascular risk factor control: AAs with a mean systolic blood pressure $<150$
\end{abstract}

$\mathrm{mm} \mathrm{Hg} \mathrm{had} 80 \%$ lower risk of early graft loss (AOR 0.2, 0.10.7 ), while low-density lipoprotein $<100 \mathrm{mg} / \mathrm{dl}$ (AOR $0.4,0.2-$ 0.8 ), triglycerides $<150 \mathrm{mg} / \mathrm{dl}$ (AOR $0.4,0.2-1.0$ ) and hemoglobin $A_{1 C}<7 \%$ (AOR 0.2, 0.1-0.6) were also protective against early graft loss in $A A$, but not in non-AA recipients. Conclusions: $A A$ recipients have a number of unique risk factors for early graft loss, suggesting that controlling cardiovascular comorbidities may be an important mechanism to reduce racial disparities in kidney transplantation.

(c) 2014 S. Karger AG, Basel

\section{Introduction}

Based on the most recent Scientific Registry of Transplant Recipients (SRTR) data, African-American (AA) kidney transplant recipients have a significantly higher risk of graft loss at 5 years post-transplant [1]. Despite over 35 years of focused research endeavors into this disparity, little has changed with this racial inequality. In the landmark 1977 analysis exposing this, Opelz and colleagues $[2,3]$ demonstrated a $10 \%$ absolute lower rate in 3 -year graft survival rates in AA recipients (25 vs. $35 \%$, $\mathrm{p}<0.001) .35$ years later, it is stubbornly similar; the 2012

\section{KARGER}

E-Mail karger@karger.com www.karger.com/ajn (c) 2014 S. Karger AG, Base

0250-8095/14/0401-0019\$39.50/0
David J. Taber, PharmD, BCPS

Division of Transplant Surgery, Medical University of South Carolina 96 Jonathan Lucas St, CSB 409 Charleston, SC 29425 (USA)

E-Mail taberd@musc.edu 
SRTR report demonstrated a 5-year absolute difference of $12 \%$ in graft survival rates between AA and non-AA patients [1]. This inequality has often been attributed to immunologic risks leading to higher acute rejection rates [4-7], socioeconomic status (SES) [8, 9], medication nonadherence $[10,11]$ and more aggressive/poorly controlled cardiovascular (CV) risk factors [12-14].

Despite a large number of studies focusing on the salient etiologies associated with the higher rates of graft loss in AA kidney transplant recipients, there continues to be significant debate into which areas to focus efforts to improve outcomes in this high-risk population [1518]. This is likely due to the contradicting and inconsistent studies published in this area of research [19-22]. Large national analyses, using registry data, lack SES and clinical data to determine true causal relationships with a number of important risks; while smaller single-center experiences usually lack sample size or long-term followup to draw meaningful conclusions [23]. Additionally, to date, there have been limited studies analyzing the effects of SES $[9-11,18]$, CV comorbidities and disease state management on racial disparities in transplantation [1215]. Our transplant program is high volume (approx. 200 kidney transplants per year) and contains a significant number of AA recipients (approx. 55\% of kidney cohort), thus having an ideal and unique set of characteristics to study racial disparities in a meaningful and clinical relevant manner. Therefore, the aim of this study was to use a large cohort of patients with detailed comprehensive data and long-term follow-up to determine if there are racial differences with the established factors associated with early graft loss in kidney transplant recipients.

\section{Materials and Methods}

\section{Study Design and Patients}

This was an IRB-approved longitudinal cohort study of renal recipients that underwent transplant between January 2005 and December 2012; 2005 was chosen as the start of this study because this is when our program began utilizing contemporary immunosuppression (tacrolimus and induction therapy) and flow cytometry in the histocompatibility laboratory. For the purposes of this study, risk and control cohorts were developed for comparison based on two primary factors: race and graft loss. Initially, groups were apportioned based on self-reported race: AA versus non-AA. Subsequently, the groups were established based on graft outcomes: the control cohort consisted of patients with ongoing graft function and at least 5 years of post-transplant follow-up (graft longevity cohort). The risk cohort consisted of patients with graft loss within 5 years post-transplant (early graft loss cohort). Patients with ongoing graft survival but follow-up of less than 5 years and patients with graft loss after 5 years were excluded from this study. The end analysis compared four unique cohorts (patients with graft longevity vs. those with early graft loss separately between AA and non-AA patients). Thus, in the end, this comparison produced a strong juxtaposition in patient types in which to compare risk factors for graft loss and do so across racial cohorts. Additional exclusion criteria included age less than 18 years at the time of transplant, recipients of non-renal solid organ transplants, or those that were lost to follow-up with inadequate clinical data available for analysis. Data was collected at baseline, from time of kidney transplant, to death, loss to follow-up or end of the study (July 2013).

\section{Outcome Measures}

The primary outcome for this study was to determine the significant factors associated with early graft loss and compare these between AA and non-AA recipients. The secondary outcomes of this study were to use multivariate models to determine independent variables that significantly influence early graft loss and compare these models across race. The final goal of the study was to determine the correlation and predictive ability of these models on determining early graft loss and compare this between AA and non-AA patients.

\section{Data Variables and Definitions}

All data was collected in a retrospective longitudinal manner from electronic and paper medical records. Initially, detailed baseline recipient and donor sociodemographics and transplant characteristics were recorded from the past medical history taken at the time of evaluation and updated at the time of transplant. All documented clinical post-transplant events were captured, including acute rejections, graft failures, and deaths. Laboratory measurements, vital signs, and clinical assessments were recorded daily for the first week post-transplant, then weekly for 1 month, then at 3 and 6 months, and finally biannually thereafter. For analyses, values were aggregated for each year independently, and then averaged across the entire follow-up time period for each patient. Graft failure was defined as a return to chronic dialysis or death. Delayed graft function was defined as the need for dialysis within 7 days following transplantation. GFRs were estimated using the fourvariable MDRD equation. The Kidney Donor Risk Index (KDRI) is a risk index which combines a number of donor factors into an aggregate score. The KDRI objectively expresses the relative risk of the graft failure for a given donor compared to the median kidney donor from the previous year. CMV infection was defined as the presence of CMV viremia of at least 2,000 copies/ml or any CMV viremia with signs and symptoms consistent with infection. BK infection was defined as BK viremia of at least 2,000 copies/ml or biopsy-proven BK nephropathy. Per institutional protocol, all PCRs were repeated for confirmation. Acute rejection was defined as biopsy-proven and at least Banff grade of 1A. Antibody-mediated rejection was biopsy-proven with $\mathrm{C} 4 \mathrm{D}+$ staining, the presence of DSA and graft dysfunction.

Immunosuppression and Anti-Infective Prophylactic Regimens

The majority of patients received induction therapy with either thymoglobulin $1.5 \mathrm{mg} / \mathrm{kg}$ i.v. daily for 3-5 doses, daclizumab $1 \mathrm{mg} /$ $\mathrm{kg}$ i.v. on days 0 and 7 post-transplant, or basiliximab $20 \mathrm{mg}$ i.v. on days 0 and 4 post-transplant. Choice of induction therapy was based on protocols that utilized thymoglobulin for high immunologic risk patients (re-transplantation, CIT $>24 \mathrm{~h}$, or PRA $>20 \%$ ), 
Fig. 1. Study flowchart displaying cohort designation and patients excluded from the analysis with reasons.

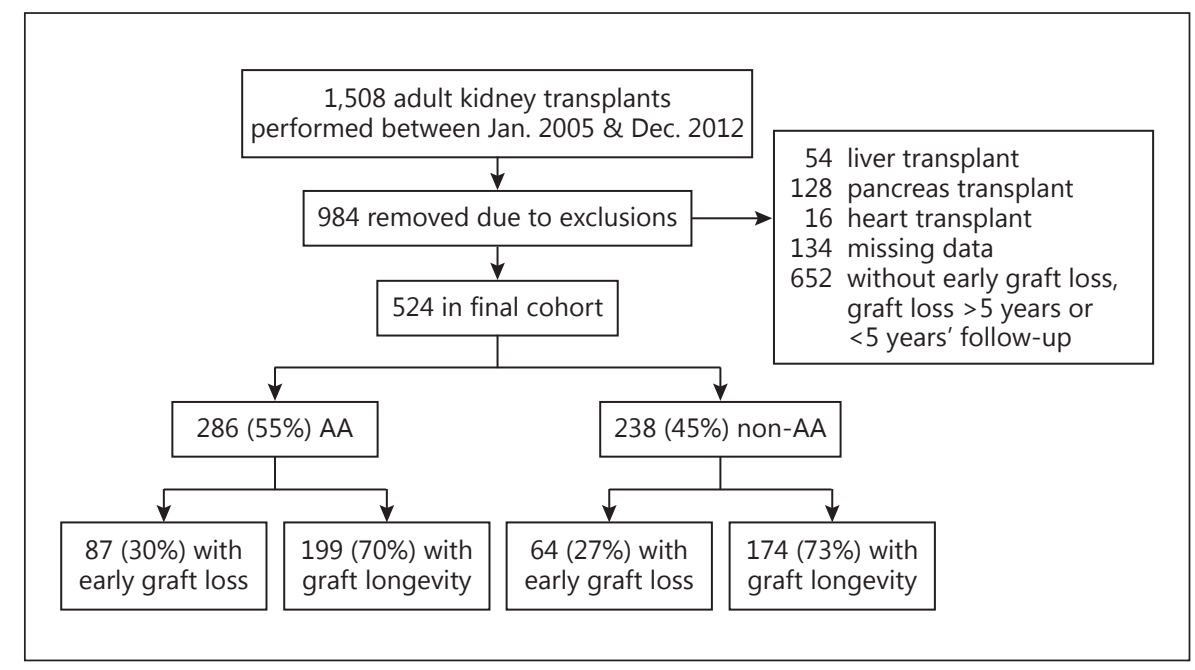

and an IL-2 receptor antibody in most other patients. Maintenance immunosuppression consisted of tacrolimus, with dose adjustments made to maintain target trough whole-blood concentrations between 8 and $12 \mathrm{ng} / \mathrm{ml}$ for weeks 1 through 6,6 to $10 \mathrm{ng} / \mathrm{ml}$ for weeks 7 through month 12 , and $>5 \mathrm{ng} / \mathrm{ml}$ after 1 year. In addition, all patients received mycophenolate mofetil $1 \mathrm{~g}$ twice daily and prednisone titrated to $5 \mathrm{mg}$ daily by day 60 post-transplant, with tapers below $5 \mathrm{mg}$ occurring rarely. Patients were converted to mTORs for CNI intolerance (nephrotoxicity being the primary etiology) and patients were converted from mycophenolate to leflunomide for BK nephropathy.

Patients at high risk of CMV $(\mathrm{D}+/ \mathrm{R}$ - or cytolytic induction therapy) received 3-6 months of valganciclovir prophylaxis depending on medication tolerability and affordability. Patients at low risk of CMV (D-/R-) did not receive CMV prophylaxis, but received herpes simplex prophylaxis with 3 months of acyclovir therapy. Moderate CMV risk patients either received 3 months of valganciclovir therapy or preemptive CMV PCR monitoring based on medication health insurance coverage and affordability. All patients received PJP prophylaxis with sulfamethoxazole/trimethoprim for 3 months and fungal prophylaxis with nystatin swishand-swallow suspension for 1 month. BK viral infection monitoring was performed by BK PCR monitoring at a minimum of 1, 3, 6,9 , and 12 months post-transplant.

\section{Statistical Analysis}

For the initial univariate analysis, patients with early graft loss (graft loss within 5 years post-transplant) were compared across racial groups for all baseline and follow-up variables. Likewise, patients with graft longevity (graft survival with at least 5 years of post-transplant follow-up) were compared in a similar fashion. All variables that demonstrated statistically significant associations on univariate analysis were included in multivariate modeling using binary logistic regression (dependent variable of early graft loss). Additional variables that were controlled for in these models included gender, age, CV history and immunologic factors (HLA mismatches, PRA, warm and cold ischemic times). Multivariate model exploration was conducted using a stepwise backward conditional approach, with variables removed at each step that lacked

Race, Cardiovascular Disease and Early Graft Loss statistical association ( $\mathrm{p}>0.2)$; model results are reported as odds ratios (ORs) and 95\% confidence intervals (CIs). Variables included in the initial model were those that demonstrated significant differences within the univariate comparisons. Model performance was assessed by developing risk probabilities for each patient to determine positive and negative predictive value, while also placing these predictive values in receiver operating characteristic (ROC) curves, with output reported in graphical form and numerically as the area under the curve (AUC, C-statistic) with 95\% CIs. Statistical significance was based on a $p$ value $<0.05$. All data was manually input into a spreadsheet (Excel, MS Office, Version 2010; Microsoft Corp., Seattle, Wash., USA) with statistical analyses performed using SPSS Version 20 (SPSS, Inc., Chicago, Ill., USA).

\section{Results}

\section{Patients}

Between January 2005 and December 2012, 1,508 adult kidney transplants were performed at our institution. Figure 1 displays the study cohort flowchart. Of the 1,508 transplants performed, 198 were excluded due to receiving extrarenal transplants (liver, pancreas, heart or lung), 134 were excluded due to missing follow-up data, and 652 were excluded due to lack of follow-up of at least 5 years or without early graft loss, leaving 524 patients in the final cohort. Of these patients, 286 (55\%) were AA and $238(45 \%)$ were non-AA. The AA cohort had 87 patients (30\%) with early graft loss (risk cohort) and 199 patients (70\%) in the control group (graft longevity), while the non-AA group had 64 patients (27\%) with early graft loss and 174 patients $(73 \%)$ with graft longevity. Mean followup for the entire cohort was $5.0 \pm 2.5$ years, which was 
similar between racial cohorts (AA $4.9 \pm 2.5$ years, nonAA $5.0 \pm 2.5$ years, $p=0.648$ ). Expectedly, the early graft loss cohort had significantly shorter follow-up compared to the graft longevity cohort ( $1.4 \pm 1.3$ vs. $6.4 \pm 0.8$ years, $\mathrm{p}<0.001$, respectively), which was consistent between racial groups (AA $1.4 \pm 1.3$ vs. $6.4 \pm 0.8$ years, $\mathrm{p}<0.001$; non-AA $1.3 \pm 1.3$ vs. $6.4 \pm 0.9$ years, $\mathrm{p}<0.001)$.

\section{Factors Associated with Early Graft Loss in Univariate} Analyses

Table 1 displays the baseline sociodemographics and transplant characteristics for patients with early graft loss versus those with graft longevity, comparing these between the AA and non-AA cohorts. For both AA and non-AA patients, three baseline factors were consistently more common in those with early graft loss, regardless of race. These included receiving disability income (OR 2.5-3.6, 95\% CI 1.8-7.0), KDRI >1.3 (OR 2.4-2.9, 95\% CI 1.1-5.5) and donors with a history of diabetes (OR $11.2-12.3,95 \%$ CI 2.4-59). AA patients had several baseline risk factors that were common in those with early graft loss (as compared to those with graft longevity), which was not demonstrated in non-AA patients; these included receiving Medicare-only health insurance (OR 3.9, 95\% CI 1.8-8.3), history of cardiac catheterization or CABG (OR 2.5, 95\% CI 1.1-5.7), not receiving induction therapy (OR 29, 95\% CI 3.6-226) and development of delayed graft function (OR 3.9, 95\% CI 1.9-8.3). Receiving peritoneal dialysis prior to transplant was more common in AA patients with graft longevity, as compared to those with early graft loss (OR $0.3,95 \%$ CI $0.1-$ 0.8 ). In the non-AA patients, unique factors that were more common in those with early graft loss (as compared to those with graft longevity) included recipients of hemodialysis prior to transplant (OR 2.3, 95\% CI 1.34.0 ) and donor history of hypertension (OR 3.8, 95\% CI $1.7-8.2)$.

Table 2 displays the immunosuppression, clinical outcomes and follow-up cardiometabolic indices for patients with early graft loss versus graft longevity, comparing these between the AA and non-AA cohorts. Three outcomes were significantly more common in those with early graft loss, regardless of race, including the development of rejection (OR 2.7-8.5, 95\% CI 1.2-83), rehospitalization (OR 2.0-4.5, 95\% CI 1.2-10.5) and BK viral infection (OR 2.5-3.4, 95\% CI 1.0-8.8). Factors that were more common in early graft loss AA patients, but not in non-AA recipients were predominantly focused around $\mathrm{CV}$ risk factor control, including having a mean followup systolic blood pressure (SBP) $<150$ mm Hg (OR 0.4,
95\% CI $0.2-0.8)$, lower mean low-density lipoprotein (LDL) (OR 0.1-0.7, 95\% CI 0.01-0.9), lower mean triglyceride (TG) level (OR 0.3-0.4, 95\% CI 0.1-0.9) and lower mean hemoglobin (Hgb) $\mathrm{A}_{1 \mathrm{C}}(\mathrm{OR} 0.2-0.4$, 95\% CI 0.11.0). In non-AA patients, unique factors more common in those with early graft loss were primarily focused on immunosuppression dosing; non-AA patients with tacrolimus trough concentrations $<8 \mathrm{ng} / \mathrm{ml}$ during the first month post-transplant had twice the risk of early graft loss (OR 2.1, 95\% CI 1.0-4.7), while patients with mean mycophenolate doses $<1,000 \mathrm{mg} /$ day after the first year post-transplant had nearly 5 times the risk of early graft loss (OR 4.6, 95\% CI 2.1-10.3).

\section{Multivariate Analysis for Factors Associated with Early Graft Loss}

Figure 2 displays the forest plots of the final multivariate model results for the dependent variable of early graft loss, separated by recipient race. Models for both AA and non-AA groups controlled for gender, age, CV history, immunologic risks (HLA mismatches, PRA, warm and cold ischemic times) and donor criteria (KDRI). CV risk factor control ( $\mathrm{HgbA}_{1 \mathrm{C}}$, TG, LDL and SBP) and two SES surrogates (income only from disability and Medicareonly health insurance) continued to be unique factors significantly associated with early graft loss in AA patients, while in non-AA patients, immunosuppression continued to be unique factors associated with early graft loss. Both rejection and infection were significant risk factors in both cohorts, regardless of race.

Figure 3 displays the ROC curves and C-statistics for the predictability of multivariate models for early graft loss, separated by race. A model containing four CV risk factor control metrics (LDL $<100 \mathrm{mg} / \mathrm{dl}$, TG $<150 \mathrm{mg} / \mathrm{dl}$, $\mathrm{SBP}<150 \mathrm{~mm} \mathrm{Hg}$ and $\mathrm{HgbA}_{1 \mathrm{C}}<7 \%$ ) was a significant predictor of early graft loss in AA patients [fig. 3a; C-statistic 0.69 (0.60-0.78), $\mathrm{p}<0.001$ ], accounting for $15 \%$ of the variability associated with early graft loss; however, a model containing these same four CV indices was not associated with early graft loss in non-AA patients [fig. 3c; C-statistic 0.57 (0.46-0.67), $p=0.211$ ], accounting for $2 \%$ of the variability associated with early graft loss. The final models for both the AA and non-AA cohorts, which included the unique risk factors described in the above section, were strong predictors of early graft loss [fig. 3b, d; C-statistic $0.81-0.83$ (0.74-0.90), p < 0.001], accounting for more than one third of the variability associated with this outcome $\left(\mathrm{R}^{2}>33 \%\right)$. In AA patients, the positive predictive value of this model in discerning patients that will develop early graft loss was $78 \%$, while the negative pre-
Taber/Douglass/Srinivas/McGillicuddy/ Bratton/Chavin/Baliga/Egede 
Table 1. Baseline donor and recipient characteristics compared between those with early graft loss versus graft longevity across racial cohorts

\begin{tabular}{|c|c|c|c|c|}
\hline \multirow[t]{2}{*}{ Baseline characteristics } & \multicolumn{2}{|c|}{$\begin{array}{l}\text { Graft loss within } 5 \text { years of } \\
\text { transplant }\end{array}$} & \multicolumn{2}{|c|}{$\begin{array}{l}\text { Graft survival of at least } 5 \text { years } \\
\text { post-transplant }\end{array}$} \\
\hline & $\mathrm{AA}(\mathrm{n}=87)$ & non-AA $(\mathrm{n}=64)$ & $\mathrm{AA}(\mathrm{n}=199)$ & non-AA $(\mathrm{n}=174)$ \\
\hline \multicolumn{5}{|l|}{ Baseline recipient sociodemographics } \\
\hline Mean age, years $( \pm S D)$ & $50 \pm 14$ & $53 \pm 15$ & $49 \pm 13$ & $51 \pm 14$ \\
\hline Female gender & $39 \%$ & $36 \%$ & $43 \%$ & $36 \%$ \\
\hline Mean BMI $( \pm S D)$ & $28 \pm 6$ & $28 \pm 6$ & $28 \pm 5$ & $27 \pm 5$ \\
\hline Did not complete high school & $16 \%$ & $14 \%$ & $8 \%$ & $7 \%$ \\
\hline Health insurance Medicare only & $24 \%$ & $13 \%$ & $8 \%$ & $10 \%$ \\
\hline Cannot read or write & $6 \%$ & $0 \%$ & $2 \%$ & $0 \%$ \\
\hline Working at the time of transplant & $10 \%$ & $18 \%$ & $16 \%$ & $26 \%$ \\
\hline Only income from disability & $41 \%$ & $39 \%$ & $22 \%$ & $15 \%$ \\
\hline Primary diagnosis diabetes & $35 \%$ & $25 \%$ & $26 \%$ & $18 \%$ \\
\hline Primary diagnosis hypertension & $37 \%$ & $20 \%$ & $34 \%$ & $13 \%$ \\
\hline \multicolumn{5}{|l|}{ Past medical history } \\
\hline Hypertension & $92 \%$ & $90 \%$ & $94 \%$ & $89 \%$ \\
\hline Diabetes & $40 \%$ & $34 \%$ & $34 \%$ & $23 \%$ \\
\hline Smoker & $22 \%$ & $33 \%$ & $20 \%$ & $29 \%$ \\
\hline Heart disease & $23 \%$ & $23 \%$ & $15 \%$ & $18 \%$ \\
\hline Hyperlipidemia & $36 \%$ & $47 \%$ & $43 \%$ & $52 \%$ \\
\hline Stroke & $10 \%$ & $3 \%$ & $10 \%$ & $5 \%$ \\
\hline Cardiac catheterization or CABG & $16 \%$ & $20 \%$ & $7 \%$ & $13 \%$ \\
\hline Acute myocardial infarction & $6 \%$ & $6 \%$ & $5 \%$ & $2 \%$ \\
\hline Congestive heart failure & $3 \%$ & $3 \%$ & $3 \%$ & $3 \%$ \\
\hline Peripheral vascular disease & $5 \%$ & $2 \%$ & $6 \%$ & $4 \%$ \\
\hline Pre-transplant dialysis & $91 \%$ & $75 \%$ & $94 \%$ & $65 \%$ \\
\hline Peritoneal dialysis & $5 \%$ & $13 \%$ & $15 \%$ & $23 \%$ \\
\hline Hemodialysis & $86 \%$ & $63 \%$ & $79 \%$ & $42 \%$ \\
\hline \multicolumn{5}{|l|}{ Baseline immunologic characteristics } \\
\hline Mean HLA mismatches $( \pm S D)$ & $5 \pm 1$ & $4 \pm 2$ & $5 \pm 1$ & $4 \pm 2$ \\
\hline Mean cold ischemic time, $\mathrm{h}( \pm \mathrm{SD})$ & $18 \pm 8$ & $16 \pm 10$ & $19 \pm 10$ & $14 \pm 11$ \\
\hline Mean warm ischemic time, $\min ( \pm \mathrm{SD})$ & $41 \pm 27$ & $37 \pm 11$ & $39 \pm 19$ & $36 \pm 9$ \\
\hline Re-transplant & $14 \%$ & $17 \%$ & $7 \%$ & $13 \%$ \\
\hline Mean \% panel reactive antibody & $9 \pm 23$ & $18 \pm 33$ & $11 \pm 23$ & $12 \pm 25$ \\
\hline$>20 \%$ & $14 \%$ & $25 \%$ & $19 \%$ & $17 \%$ \\
\hline$>80 \%$ & $6 \%$ & $11 \%$ & $5 \%$ & $6 \%$ \\
\hline \multicolumn{5}{|l|}{ Baseline donor characteristics } \\
\hline Mean donor KDRI $( \pm S D)$ & $1.34 \pm 0.41$ & $1.35 \pm 0.42$ & $1.14 \pm 0.33$ & $1.18 \pm 0.36$ \\
\hline \multicolumn{5}{|l|}{ Donor type } \\
\hline Living donor & $7 \%$ & $19 \%$ & $11 \%$ & $33 \%$ \\
\hline Extended criteria & $14 \%$ & $22 \%$ & $9 \%$ & $13 \%$ \\
\hline Deceased after cardiac death & $0 \%$ & $0 \%$ & $1 \%$ & $2 \%$ \\
\hline Female gender & $48 \%$ & $48 \%$ & $40 \%$ & $46 \%$ \\
\hline African-American & $32 \%$ & $19 \%$ & $34 \%$ & $14 \%$ \\
\hline \multicolumn{5}{|l|}{ Past medical history } \\
\hline Hypertension & $23 \%$ & $39 \%$ & $17 \%$ & $14 \%$ \\
\hline Diabetes & $12 \%$ & $18 \%$ & $1 \%$ & $2 \%$ \\
\hline Died due to stroke & $44 \%$ & $45 \%$ & $30 \%$ & $32 \%$ \\
\hline Hepatitis C positive & $5 \%$ & $0 \%$ & $0 \%$ & $2 \%$ \\
\hline \multicolumn{5}{|l|}{ Perioperative factors } \\
\hline \multicolumn{5}{|l|}{ Induction therapy } \\
\hline None/not documented & $13 \%$ & $5 \%$ & $0 \%$ & $2 \%$ \\
\hline IL-2 receptor antibody & $52 \%$ & $52 \%$ & $62 \%$ & $65 \%$ \\
\hline Cytolytic therapy & $35 \%$ & $44 \%$ & $38 \%$ & $34 \%$ \\
\hline Delayed graft function & $23 \%$ & $9 \%$ & $7 \%$ & $5 \%$ \\
\hline
\end{tabular}


Table 2. Immunosuppression and clinical outcomes compared between those with early graft loss versus graft longevity across racial cohorts

\begin{tabular}{|c|c|c|c|c|}
\hline \multirow[t]{2}{*}{ Post-transplant outcomes } & \multicolumn{2}{|c|}{$\begin{array}{l}\text { Graft loss within } 5 \text { years of } \\
\text { transplant }\end{array}$} & \multicolumn{2}{|c|}{$\begin{array}{l}\text { Graft survival of at least } 5 \text { years } \\
\text { post-transplant }\end{array}$} \\
\hline & $\mathrm{AA}(\mathrm{n}=87)$ & non-AA $(\mathrm{n}=64)$ & $\mathrm{AA}(\mathrm{n}=199)$ & non-AA $(\mathrm{n}=174)$ \\
\hline \multicolumn{5}{|l|}{ Immunologic characteristics } \\
\hline \multicolumn{5}{|c|}{ Mean tacrolimus trough concentrations } \\
\hline First week & $7.7 \pm 2.2$ & $8.6 \pm 2.6$ & $7.4 \pm 2.6$ & $9.0 \pm 2.5$ \\
\hline First month & $8.4 \pm 1.7$ & $8.7 \pm 2.0$ & $8.5 \pm 1.8$ & $9.5 \pm 1.7$ \\
\hline First year & $8.3 \pm 1.5$ & $8.5 \pm 1.8$ & $8.5 \pm 1.4$ & $8.9 \pm 1.3$ \\
\hline After first year & $6.7 \pm 2.7$ & $6.0 \pm 2.5$ & $6.8 \pm 1.8$ & $6.6 \pm 1.8$ \\
\hline \multicolumn{5}{|l|}{ Mean mycophenolate doses } \\
\hline First month & $1,603 \pm 490$ & $1,466 \pm 508$ & $1,652 \pm 462$ & $1,534 \pm 419$ \\
\hline Three months & $1,498 \pm 573$ & $1,271 \pm 598$ & $1,581 \pm 511$ & $1,465 \pm 478$ \\
\hline After first year & $1,327 \pm 710$ & $885 \pm 786$ & $1,452 \pm 582$ & $1,371 \pm 551$ \\
\hline Acute cellular rejection & $35 \%$ & $17 \%$ & $16 \%$ & $6 \%$ \\
\hline Within 3 months & $15 \%$ & $6 \%$ & $7 \%$ & $2 \%$ \\
\hline Within 6 months & $17 \%$ & $8 \%$ & $8 \%$ & $2 \%$ \\
\hline Within 1 year & $24 \%$ & $13 \%$ & $9 \%$ & $3 \%$ \\
\hline Severe rejection $($ Banff $\geq 1 \mathrm{~B})$ & $26 \%$ & $11 \%$ & $9 \%$ & $2 \%$ \\
\hline Acute antibody-mediated & $14 \%$ & $5 \%$ & $6 \%$ & $1 \%$ \\
\hline Borderline & $12 \%$ & $20 \%$ & $15 \%$ & $13 \%$ \\
\hline \multicolumn{5}{|l|}{ Readmissions } \\
\hline 30 days & $28 \%$ & $23 \%$ & $11 \%$ & $6 \%$ \\
\hline 90 days & $41 \%$ & $36 \%$ & $18 \%$ & $12 \%$ \\
\hline 1 year & $58 \%$ & $45 \%$ & $30 \%$ & $24 \%$ \\
\hline Any & $70 \%$ & $58 \%$ & $54 \%$ & $36 \%$ \\
\hline Readmitted for CV event & $12 \%$ & $22 \%$ & $8 \%$ & $8 \%$ \\
\hline Readmitted for infectious event & $22 \%$ & $22 \%$ & $21 \%$ & $15 \%$ \\
\hline Readmitted for surgical issue & $16 \%$ & $9 \%$ & $10 \%$ & $10 \%$ \\
\hline \multicolumn{5}{|l|}{ Infections } \\
\hline Cytomegalovirus & $15 \%$ & $6 \%$ & $12 \%$ & $8 \%$ \\
\hline $\mathrm{BK}$ virus & $10 \%$ & $9 \%$ & $7 \%$ & $6 \%$ \\
\hline Any significant infection & $45 \%$ & $36 \%$ & $34 \%$ & $27 \%$ \\
\hline Convert to mTOR & $10 \%$ & $12 \%$ & $15 \%$ & $13 \%$ \\
\hline \multicolumn{5}{|l|}{ Cardiometabolic characteristics } \\
\hline Mean protein/creatinine ratio & $1.17 \pm 3.69$ & $1.23 \pm 4.53$ & $0.70 \pm 1.27$ & $0.43 \pm 0.81$ \\
\hline Mean systolic blood pressure & $140 \pm 15$ & $134 \pm 12$ & $136 \pm 15$ & $134 \pm 14$ \\
\hline Mean diastolic blood pressure & $79 \pm 13$ & $74 \pm 9$ & $77 \pm 8$ & $76 \pm 8$ \\
\hline Mean low-density lipoproteins ${ }^{+}$ & $109 \pm 38$ & $83 \pm 31$ & $96 \pm 25$ & $95 \pm 53$ \\
\hline Mean triglycerides $^{+}$ & $143 \pm 71$ & $165 \pm 78$ & $120 \pm 60$ & $163 \pm 78$ \\
\hline Mean hemoglobin $\mathrm{A}_{1 \mathrm{C}}{ }^{+, *}$ & $7.8 \pm 2.5$ & $7.1 \pm 1.8$ & $7.5 \pm 1.6$ & $6.8 \pm 1.2$ \\
\hline \multicolumn{5}{|l|}{ Compelling medication use } \\
\hline Antiplatelet therapy & $41 \%$ & $34 \%$ & $53 \%$ & $44 \%$ \\
\hline$\beta$-Blocker & $51 \%$ & $44 \%$ & $58 \%$ & $51 \%$ \\
\hline ACE inhibitor or ARB & $39 \%$ & $36 \%$ & $54 \%$ & $46 \%$ \\
\hline Statin therapy & $43 \%$ & $34 \%$ & $53 \%$ & $49 \%$ \\
\hline Other anti-lipid therapy & $37 \%$ & $28 \%$ & $43 \%$ & $43 \%$ \\
\hline
\end{tabular}

${ }^{+}$Values represent the mean during the entire post-transplant follow-up period.

* This is reported only for patients with a diagnosis of diabetes. 
BK viral infection

Acute rejection

History of cardiac catheterization

Health insurance Medicare only

Income from disability only

Mean $\mathrm{HgbA}_{1 \mathrm{c}}<7 \%$

Mean TG $<150 \mathrm{mg} / \mathrm{dl}$

Mean LDL $<100 \mathrm{mg} / \mathrm{dl}$

Mean SBP $<150 \mathrm{~mm} \mathrm{Hg}$

a

Living donor

Mean FK level $<8 \mathrm{ng} / \mathrm{ml}$ during 1st month Readmitted for infection

Mean MMF dose $<1,000 \mathrm{mg} /$ day $>1$ st year

Acute rejection

Income from disability only

b
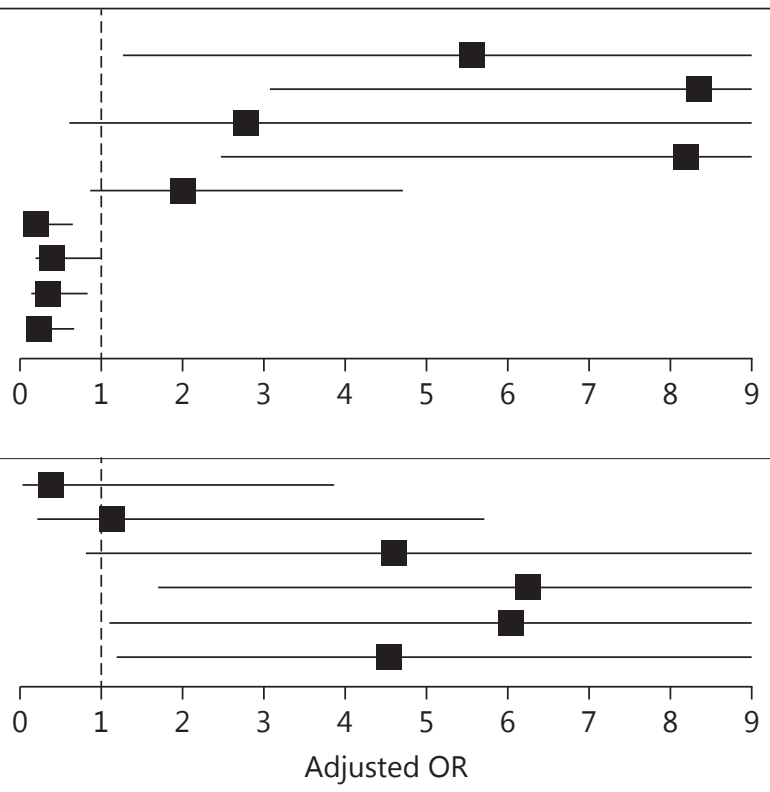

Fig. 2. Forest plots of the final multivariate model results for the dependent variable of early graft loss. a AA patients. b Non-AA patients.

dictive value in discriminating which patients will not have graft loss within 5 years post-transplant was $88 \%$. In non-AA patients, the positive predictive value was $60 \%$ and the negative predictive value was $88 \%$.

\section{Discussion}

Despite years of focused research in racial disparities, AA kidney transplant recipients continue to experience a disproportionately higher rate of graft loss [1] and the predominant factors driving this disparity are heavily debated. The reasons for this continued controversy are likely related to the complex interacting etiologies converging to create this disparity, coupled with numerous studies that provide contradicting findings [15-17]. The results of the analysis presented in this paper provide detailed and comprehensive assessment of baseline and follow-up clinical data that cannot be captured in national registry studies [23]. The results demonstrate that AA patients have unique baseline and follow-up characteristics that predispose them to graft loss. In particular, it appears that surrogates of baseline SES (Medicare-only insurance and disability-only income), coupled with CV risk factor control during the post-transplant follow-up period provide strong associations with graft loss. Other than disability income, these factors were unique to AA recipients, and did not appreciably influence early graft loss in the nonAA cohort. Taken in their entirety, these results provide evidence to potentially support interventional studies aimed at improving CV risk factor control as a means to diminish racial disparities in kidney transplantation.

The finding that the development of rejection was a consistent risk factor for early graft loss in both AA and non-AA patients is an expected result $[24,25]$. Given the consistent predictability of rejection on graft loss across all racial groups, future interventions should focus on methods to reduce rejection to optimize graft outcomes in all patients, and given the additional unique risk factors in AA patients identified in this study, it is unlikely that solely focusing on reducing rejection will produce robust improvements in racial disparities in transplantation. It is interesting to note that in the univariate analysis, lack of induction therapy was a strong risk factor for early graft loss in AA recipients, while cytolytic induction was a risk factor for graft loss in non-AA patients. This data supports evidence from previous studies, suggesting AA patients may obtain more benefit from potent induction therapy [16]. A recently completed prospective study from our institution demonstrated the AA patients likely benefited more than non-AA from antithymocyte globulin induction by reducing acute rejection rates, even with- 


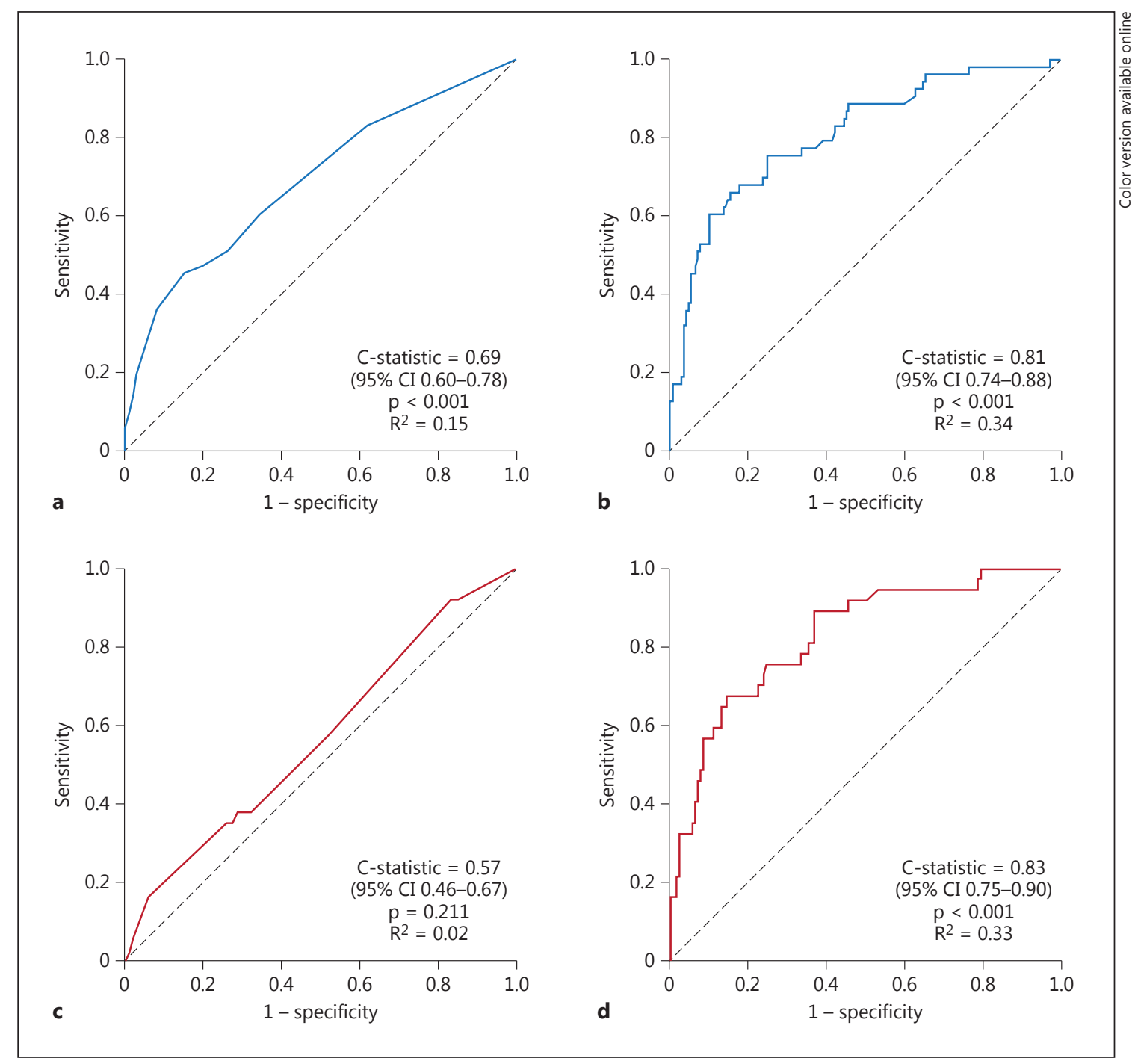

Fig. 3. ROC curves displaying the association between various post-transplant factors and early graft loss for both AA patients (a, b) and non-AA patients (c, d). Models containing four CV risk factor control metrics (LDL $<100 \mathrm{mg} / \mathrm{dl}, \mathrm{TG}<150 \mathrm{mg} / \mathrm{dl}, \mathrm{SBP}<150$ $\mathrm{mm} \mathrm{Hg} \mathrm{HgbA}_{1 \mathrm{C}}<7 \%$ ) was a significant predictor of early graft loss in AA patients [a; C-statistic $0.69(0.60-0.78), \mathrm{p}<0.001$ ], while a model containing these same four $\mathrm{CV}$ metrics was not associated with early graft loss in non-AA patients [c; C-statistic $0.57(0.46-$ $0.67), \mathrm{p}=0.211]$. The final models for both the AA and non-AA cohorts, which included the risk factors demonstrating statistical significance in tables 1 and 2, were strong predictors of early graft loss [b, d; C-statistic 0.81-0.83 (0.74-0.90), p < 0.001]. in the context of a potent contemporary triple-drug maintenance immunosuppressant regimen of tacrolimus, mycophenolate and prednisone [26].

The development of post-transplant infection was also a consistent risk factor for early graft loss, regardless of race. Given the abundance of previous studies demonstrating the risks of infection on graft loss, this is also an expected finding. Focusing efforts on reducing the impact of infection or BK virus on graft loss will likely improve outcomes, but given the consistent effect of this on graft loss across both AA and non-AA patients, this would not likely reduce racial disparities $[27,28]$.

Previous studies have demonstrated similar results to the data presented in this analysis with regard to a number of risk factors particularly important in AA kidney transplant recipients. These include SES factors [8-11] and post-transplant hypertension control [12]. Although modifying SES is a difficult, improving post-transplant 
CV risk factor control is certainly obtainable [15]. Chisholm et al. [29] demonstrated improved hypertension control in AA patients with interventions focused on medication adherence and cost. However, within the transplant population, there is paucity in literature demonstrating improved patient or graft survival through enhanced CV risk factor control. Certainly, given the strong body of literature within the general population [30-32] and the fact that transplant patients usually have multiple CV risk factors, improving hypertension, dyslipidemia and diabetes management in transplant recipients should be expected to improve graft and patient survival $[32,34]$. An important question, which the results of the data presented in this study cannot answer, is what is the best mechanism to optimize CV risk factor control in transplant patients, and in particular AA transplant recipients? It is unlikely that a single strategy to this complex issue will produce robust results. Rather, a multidimensional intervention that focuses on compelling medication prescribing and dosing optimization, coupled with improving adherence and healthy lifestyle choices leading to weight management and exercise regimens may be the best approach to obtain enduring results $[32,35]$.

There are several important limitations to this study that are worthy of discussion. First, this was a retrospective study; limitations with retrospective cohort studies include the potential for selection bias, recall misclassification and missing data. Data collection occurred through the use and validation of both electronic and paper medical records to minimize these potential limitations. An additional limitation to the retrospective study design includes lacking the ability to prospectively randomize and risk-stratify non-modifiable donor and recipient characteristics at the study onset; however, multivariate modeling was used to minimize this limitation. Although this study contained a large number of patients with significant follow-up, its single-center design did limit study power, and certainly separating groups by both graft loss and race also decreased the studies' overall power. However, these limitations are somewhat offset by the fact that detailed baseline SES and medical histories were captured in this study. Additionally, thorough follow-up clinical data, including blood pressures, serum lipids and $\mathrm{HgbA}_{1 \mathrm{C}}$ s were collected and included in this analysis, which is lacking from previous analyses in this area of research. Baseline and follow-up data, such as education level, was included in this analysis, which would not be easily obtainable in a multicenter retrospective study or one that utilizes national registry data from the SRTR [23]. Finally, it should be noted that although limiting the time frame of this study from the beginning in 2005 may have limited the sample size and reduced study power, it did produce a study cohort that is well representative of contemporary kidney transplant recipients, including age, medical comorbidities, donor risk and immunosuppression regimens.

In conclusion, the results of this analysis demonstrate that in a contemporary cohort of kidney transplant recipients, acute allograft rejection and infection continue to be prominent risk factors associated with graft loss. Within AA recipients, SES and CV risk factor control are significant and unique factors associated with early graft loss. Future studies should focus interventions on modifying these factors as a mechanism to improve graft outcomes in this high-risk cohort of kidney transplant recipients.

\section{Acknowledgements}

The research reported in this publication was supported by the National Institute of Diabetes and Digestive and Kidney Diseases of the National Institutes of Health under award Nos. K23DK099440 and T35 DK007431.

\section{Disclosure Statement}

The authors have no conflicts of interest to disclose.

\section{References}

Race, Cardiovascular Disease and Early Graft Loss
1 Organ Procurement and Transplantation Network (OPTN) and Scientific Registry of Transplant Recipients (SRTR): OPTN/SRTR 2010 Annual Data Report. Rockville/MD, Department of Health and Human Services, Health Resources and Services Administration, Healthcare Systems Bureau, Division of Transplantation, 2011.

-2 Opelz G, Mickey MR, Terasaki PI: Influence of race on kidney transplant survival. Transplant Proc 1977;9:137-142.
-3 Terasaki PI, Opelz G, Mickey MR: Summary of kidney transplant data, 1977 - factors affecting graft outcome. Transplant Proc 1978; 10:417-421.

-4 Ciancio G, Burke GW, Suzart K, Mattiazzi A, Vaidya A, Roth D, Kupin W, Rosen A, Johnson N, Miller J: The use of daclizumab, tacrolimus and mycophenolate mofetil in AfricanAmerican and Hispanic first renal transplant recipients. Am J Transplant 2003;3:10101016. 
5 Neylan JF: Racial differences in renal transplantation after immunosuppression with tacrolimus versus cyclosporine. FK506 kidney transplant study group. Transplantation 1998;27:65:515-523.

-6 Podder H, Podbielski J, Hussein I, Katz S, Buren C, Kahan BD: Sirolimus improves the two-year outcome of renal allografts in African-American patients. Transplant Int 2001 14:135-142.

$\checkmark 7$ Weber M, Deng S, Arenas J, Aradhye S, Grossman R, Shaw L, Naji A, Barker C, Brayman KL: Decreased rejection episodes in African-American renal transplant recipients receiving mycophenolate mofetil/tacrolimus therapy. Transplant Proc 1997;29:3669-3670.

-8 Butkus DE, Meydrech EF, Raju SS: Racial differences in the survival of cadaveric renal allografts. Overriding effects of HLA matching and socioeconomic factors. N Engl J Med 1992;327:840-845.

$\checkmark 9$ Curtis JJ: Kidney transplantation: racial or socioeconomic disparities? Am J Kidney Dis 1999;34:756-758.

-10 Schweizer RT, Rovelli M, Palmeri D, Vossler E, Hull D, Bartus S: Noncompliance in organ transplant recipients. Transplantation 1990; 49:374-377.

11 Kalil R, Heim-Duthoy K, Kasiske B: Patients with a low income have reduced renal allograft survival. Am J Kidney Dis 1992;20:63.

12 Cosio FG, Dillon JJ, Falkenhain ME, Tesi RJ, Henry ML, Elkhammas EA, Davies EA, Bumgardner GL, Ferguson RM: Racial differences in renal allograft survival: the role of systemic hypertension. Kidney Int 1995;47:1136-1141.

$\checkmark 13$ Cosio FG, Pesavento TE, Kim S, Osei K, Henry $\mathrm{M}$, Ferguson RM: Patient survival after renal transplantation. IV. Impact of post-transplant diabetes. Kidney Int 2002;62:14401446.

14 Cosio FG, Hickson LJ, Griffin MD, Stegall MD, Kudva Y: Patient survival and cardiovascular risk after kidney transplantation: the challenge of diabetes. Am J Transplant 2008; 8:593-599.

15 Young CJ, Kew C: Health disparities in transplantation: focus on the complexity and challenge of renal transplantation in AfricanAmericans. Med Clin N Am 2005;89:10031031.
16 Malat GE, Culkin C, Palya A, Ranganna K, Kumar MS: African-American kidney transplantation survival: the ability of immunosuppression to balance the inherent pre- and post-transplant risk factors. Drugs 2009;69: 2045-2062.

17 Young CJ, Gaston RS: African-Americans and renal transplantation: disproportionate need, limited access, and impaired outcomes. Am J Med Sci 2002;323:94.

18 Butkus DE, Meydrech EF, Raju SS: Racial differences in the survival of cadaveric renal allografts. Overriding effects of HLA matching and socioeconomic factors. N Engl J Med 1992;327:840-845.

19 Navaneethan SD, Singh S: A systematic review of barriers in access to renal transplantation among African-Americans in the United States. Clin Transplant 2006;20:769-775.

20 Pallet N, Thervet E, Alberti C, Emal-Aglaé V, Bedrossian J, Martinez F, Roy C, Legendre C: Kidney transplant in Black recipients: are African-Europeans different from AfricanAmericans? Am J Transplant 2005;5:26822687.

21 Yeates K, Wiebe N, Gill J, Sima C, Schaubel D, Holland D, Hemmelgarn B, Tonelli M: Similar outcomes among Black and White renal allograft recipients. J Am Soc Nephrol 2009; 20:172-179.

22 Chakkera HA, O’Hare AM, Johansen KL, Hynes D, Stroupe K, Colin PM, Chertow GM: Influence of race on kidney transplant outcomes within and outside the Department of Veterans Affairs. J Am Soc Nephrol 2005;16: 269-277.

23 Kaplan B, Schold J, Meier-Kriesche HU: Overview of large database analysis in renal transplantation. Am J Transplant 2003;3: 1052-1056.

24 Schold JD, Srinivas TR, Braun WE, et al: The relative risk of overall graft loss and acute rejection among African-American renal transplant recipients is attenuated with advancing age. Clin Tranpslant 2011;25:721-730.

25 Sellares J, de Freitas DG, Mengel M, et al: Understanding the causes of kidney transplant failure: the dominant role of antibody-mediated rejection and nonadherence. Am J Transplant 2012;12:388-399.
26 Pilch NA, Taber DJ, Moussa O, et al: Prospective randomized controlled trial of rabbit antithymocyte globulin with IL-2 receptor antagonist induction therapy in kidney transplantation. Ann Surg 2014;259:888-893.

27 Binet I, Volker N, Hirsch HH, et al: Polyomavirus disease under new immunosuppressive drugs: a cause of renal graft dysfunction and graft loss. Transplantation 1999;67:918-922.

28 Johnston O, Jaswal D, Gill JS, et al: Treatment of polyomavirus infection in kidney transplant recipients: a systematic review. Transplantation 2010;89:1057-1070.

29 Chisholm MA, Mulloy LL, Jagadeesan M, Martin BV, DiPiro JT: Effect of clinical pharmacy services on the blood pressure of African-American renal transplant patients. Ethn Dis 2002; 12:392-397.

30 Brugts JJ, Yetgin T, Hoeks SE, et al: The benefits of statins in people without established cardiovascular disease with cardiovascular risk factors: meta-analysis of randomised controlled trials. Br Med J 2009;338:23762383.

31 Kelly TN, Bazzano LA, Fonseca VA, et al: Systematic review: glucose control and cardiovascular disease in type 2 diabetes. Ann Intern Med 2009;151:394-403.

32 Thompson AM, Hu T, Eshelbrenner CL, et al: Antihypertensive treatment and secondary prevention of cardiovascular disease events among person without hypertension. JAMA 2011;305:913-922.

33 Carpenter MA, Weir MR, Adey DB, House AA, Bostom AG, Kusek JW: Inadequacy of cardiovascular risk factor management in chronic kidney transplantation - evidence from the FAVORIT study. Clin Transplant 2012;26:E438-E446.

34 Pilmore HL, Skeans MA, Snyder JJ, Israni AK, Kasiske BL: Cardiovascular disease medications after renal transplantation: results from the patient outcomes in renal transplantation study. Transplantation 2011;91:542-551.

35 Taber DJ, Pilch NA, Meadows HB, McGillicuddy JW, Bratton CF, Chavin KD, Baliga PK, Egede, LE: The impact of cardiovascular disease and risk factor control on ethnic disparities in kidney transplant. J Cardiovasc Pharm Ther 2013;18:243-250. 